\title{
Impending relapse of myelodysplastic syndrome after allogeneic transplant is difficult to diagnose and requires a multi- modal approach
}

\author{
Elizabeth L. Courville ${ }^{1 *}$ (D) Megan Griffith', Celalettin Ustun², Sophia Yohe ${ }^{1}$ and Erica Warlick²
}

\begin{abstract}
Background: The only potentially curative therapy for myelodysplastic syndrome is allogeneic hematopoietic cell transplant; unfortunately, there is a high relapse rate. The objective of this study was to perform a detailed clinicopathologic study of patients with relapsed myeloid neoplasm following allogeneic hematopoietic cell transplant for myelodysplastic syndrome.

Methods: Pre-transplant, post-transplant, and relapse bone marrow and peripheral blood morphologic features (including dysplasia) were retrospectively evaluated by study authors. Clinical features and results of cytogenetic analysis and engraftment/chimerism studies were obtained from the medical record.

Results: Our study describes 21 patients with a median time to relapse of 6 months (range 2-82). Ten of the patients relapsed with higher grade disease, including six with overt acute myeloid leukemia. Pre-transplant megakaryocyte dysplasia was associated with dysplastic megakaryocytes in the relapse specimen; however, neither erythroid dysplasia nor granulocytic dysplasia were associated with their counterpart in the relapse specimen. Relapse specimens had a lower marrow cellularity and higher blast percentage than pre-transplant disease. Cytogenetic comparisons before and after transplant showed variety, including clonal evolution (22\%), the same abnormal clone (33\%), or a different abnormal clone (22\%).

Conclusions: Our detailed review of post-transplant marrow biopsies prior to relapse highlights the difficulty in diagnosing relapse and particularly impending relapse.
\end{abstract}

Keywords: Allogeneic stem cell transplant, Myelodysplastic syndrome, Relapse, Cytogenetic, Acute myeloid leukemia

\section{Background}

Myelodysplastic syndromes (MDS) are the most commonly diagnosed myeloid neoplasms in the United States. MDS are clonal hematopoietic neoplasms characterized by ineffective hematopoiesis and varying risk of transformation to acute myeloid leukemia (AML). According to the World Health Organization (WHO) [1], MDS are further subclassified based on the number of dysplastic lineages, the number of cytopenic lineages, the percentage of ring sideroblasts, the bone marrow

\footnotetext{
* Correspondence: ecourvil@umn.edu

'Department of Laboratory Medicine and Pathology, University of Minnesota, 420 Delaware St SE, MMC 609, Minneapolis, MN 55455, USA

Full list of author information is available at the end of the article
}

and peripheral blood blast percentages, the presence or absence of Auer rods, and cytogenetic findings. This heterogeneous group has variable prognoses and treatments ranging from supportive care only to chemotherapy (hypomethylating agent based therapy or intensive AML-type induction chemotherapy) to possible subsequent allogeneic hematopoietic cell transplant (HCT) [2, 3]. Therapy choice is guided by risk stratification based on the International Prognostic Scoring System (IPSS) and revised IPSS (IPSS-R) [4, 5], as well as other patient factors including age, performance status, transfusion needs, and response to first-line therapy, and donor options. 
The only potentially curative therapy for MDS is allogeneic HCT. Unfortunately, relapse remains a concern with rates in the $20-50 \%$ range [6-8]. The relapse risk likely depends on multiple factors including the preparative regimen (myeloablative versus non-myeloablative or reducedintensity), stem cell and donor source (umbilical cord blood versus sibling or adult unrelated bone marrow/peripheral blood), and pre-transplant MDS disease burden as well as MDS disease characteristics. The optimal method or combination of methods to detect impending relapse following transplant is not clear nor is the optimal therapeutic intervention for impending relapse [7].

In this study, we performed a detailed assessment of 21 patients with relapsed myeloid neoplasm following allogeneic HCT. We evaluated diagnostic MDS characteristics (IPSS, WHO classification, cytogenetics) as well as a comparison of pre-transplant and relapsed disease morphology, cytogenetics, and flow cytometry. Intervening (post-transplant, pre-relapse) data was also reviewed. Late relapse cases arising $>6$ months after transplant were compared to early relapse cases.

\section{Methods}

This retrospective study was approved by the University of Minnesota Institutional Review Board (Study Number 1312 M46725) and performed according to the ethical standards of our institution. As part of the approval process by the University of Minnesota IRB, it was determined that informed consent was not required for this retrospective research study. Patients were identified by search of the bone marrow transplant database. Adult patients ( $\geq 18$ years old) were included if they received their first allogeneic HCT for MDS at the University of Minnesota between 2000 and 2015. Patients were excluded if their pre-transplant bone marrow biopsy slides were not reviewed at our institution, if no post-transplant biopsy was obtained, or if there was persistent marrow disease post-transplant. The electronic medical record was used to extract clinical information. Pathology reports from the following specimens were reviewed for all patients: [1] original MDS diagnosis, [2] pre-transplant MDS diagnosis, [3] immediate pre-transplant biopsy (following cytoreductive therapy), [4] post-transplant biopsy specimens interpreted at our institution up to and including [5] the post-transplant relapse biopsy. The information extracted included the bone marrow cellularity and blast percentage, peripheral blood counts, and circulating blast percentages. The post-transplant cases, [4], were categorized as "negative" or "indeterminate" for morphologic evidence of myeloid neoplasm based on review of the original pathology report.

Available slides from the pre-transplant MDS diagnosis [2] (slides available for 15 patients) and posttransplant relapse marrow [5] (slides available for 20 patients) were reviewed by study author EC and scored for dysplasia in a semi-quantitative manner modified from the system used by Weinberg, et al. [9]. Reviewed slides included H\&E stained slides of the trephine core, immunohistochemical stains performed at the time of diagnosis, Wright-Giemsa stained marrow aspirate and peripheral blood slides, and Dacie (iron) stained marrow slides. Dysplasia was scored in each lineage in increments of $10 \%$. Specific dysplastic features in the megakaryocyte lineage were: micromegakaryocytes, hypolobated or monolobated megakaryocytes of normal size, megakaryocytes with two or more separated rounded nuclear lobes. Specific dysplastic features in the erythroid lineage were: megaloblastoid change, multinucleation, nuclear irregularities, pyknosis, and basophilic stippling. Ring sideroblast percentage was documented. Specific dysplastic features in the granulocyte lineage were: abnormal nuclear shape and hypogranulation. A lineage was only evaluated if sufficient cells were available for analysis.

To evaluate for morphologic features of impending relapse, slides from the bone marrow biopsy specimen immediately prior to the relapse specimen were reviewed by study author SY, who was blinded to the original pathology interpretation and the results of corresponding ancillary studies. The presence or absence of dysplasia, and affected lineages, was documented, and blasts were evaluated as increased or decreased. Based on the morphology alone, the specimens were re-interpreted as "negative", "positive", or "indeterminate" for relapsed myeloid neoplasm.

Flow cytometric studies and cytogenetic analysis were performed using standard techniques at the time of diagnosis, with the interpretive reports reviewed for this study. Flow cytometry studies evaluated for aberrant antigen expression on myeloid blasts using either 4color or 10-color panels. Antigens evaluated included CD3, CD7, CD10, CD13, CD14, CD15, CD19, CD33, CD34, CD45, CD56, CD117, and HLA-DR. Myeloid maturation patterns by flow cytometry were not evaluated. Engraftment/chimerism analysis was performed as previously described [10], with interpretive reports reviewed for this study.

Statistical analysis was performed using the IBM SPSS Statistics program version 22. A two-tailed Fisher's exact test was used for categorical data and a MannWhitney U test (for independent samples) or Wilcoxon signed-ranks test (for related samples) was used for continuous data.

\section{Results}

Our patient cohort included 10 males and 11 females. The median age at transplant was 59 years (range 34 to 71). Three patients had a medication history compatible with therapy-related myeloid neoplasm. One patient 
received cyclophosphamide for scleroderma/interstitial lung disease for 2 years ending 3 years prior to MDS diagnosis, one patient received multi-agent chemotherapy for breast cancer (including paclitaxel, doxorubicin, and cyclophosphamide) 4 years prior to MDS diagnosis, and one patient was treated with doxorubicin and cyclophosphamide for breast cancer 10 years prior to MDS diagnosis.

Eighteen patients were treated with a non-myeloablative/ reduced intensity conditioning regimen prior to transplant (cytarabine, fludarabine, and total body irradiation with or without anti-thymocyte globulin, $n=17$, or busulfan/fludarabine, $n=1$ ) and three were treated with a myeloablative conditioning regimen (busulfan and cytarabine or cytarabine and fractionated total body irradiation). Graft-versushost-disease prophylaxis included cyclosporine and mycophenolate mofetil $(n=17)$, cyclosporine and methotrexate $(n=3)$ or methotrexate and tacrolimus $(n=1)$. Source of donor stem cells included umbilical cord blood $(n=10)$, sibling $(n=9)$, and unrelated donor $(n=2)$.

\section{Pre-transplant MDS versus relapse characteristics}

Patient were most commonly transplanted for refractory anemia with excess blasts (RAEB-1 or 2, $n=13$ ) and refractory cytopenias with multilineage dysplasia (RCMD, $n=3$ ). In eight patients, disease prior to transplant included ring sideroblasts, with five having a ring sideroblast percentage $\geq 15 \%$. Of the relapses, six cases relapsed with overt AML and one additional case (Case 75) progressed to AML 2 months after initial relapse (only withdrawal of immunosuppression prior to progression). Two cases relapsed as MDS with increased blasts where the pre-transplant disease lacked excess blasts, and an additional case lacked an increase in blasts in both the pre-transplant and relapse specimens but progressed to RAEB-1 2 months after initial relapse despite a reduction in immunosuppression in the intervening time.

The presence of dysplastic megakaryocytes in the pretransplant specimen showed a statistically significant association with the presence of dysplastic megakaryocytes in the relapse specimen $(p=0.018)$ with increased significance $(p=0.001)$ when a dysplastic megakaryocyte threshold of $50 \%$ was applied. In contrast, neither erythroid dysplasia/ring sideroblasts in the pre-transplant specimen nor granulocytic dysplasia in the pre-transplant specimen was associated with their counterpart in the relapse specimen ( $p=0.520 / 1.0$, and 0.070 , respectively).

There was a significant difference between the pretransplant and relapse bone marrow cellularity [pretransplant median of $73 \%$ (range 15-95\%) and relapse median of $45 \%$ (range $10-90 \%$ ), $p=0.003$ ] and blast percentage [pre-transplant median of 5\% (range 1-19\%) and relapse median of $10 \%$ (range $0.2-51 \%$ ), $p=0.023$ ] but there was no significant difference between the pretransplant and relapse peripheral blood blast percentage.
There was a significant difference between the pretransplant and relapse platelet count [pre-transplant median of $73 \times 10^{9} / \mathrm{L}$ (range $5-1242$ ) and relapse median $38 \times 10^{9} / \mathrm{L}$ (range $7-189$ ), $p=0.028$ ] and a borderline significant difference between the pre-transplant and relapse white blood cell count [pre-transplant median $3.3 \times 10^{9} / \mathrm{L}\left(1-33\right.$ range) and relapse median $2.5 \times 10^{9} / \mathrm{L}$ (0.6-7.6 range) $p=0.054$ ]. No statistically significant difference was seen between the pre-transplant and relapse hemoglobin, mean corpuscular volume (MCV) or absolute neutrophil count.

The majority $(18 / 21,86 \%)$ of cases had an abnormal karyotype in the myeloid neoplasm before and/or after transplant. Pre- and post-transplant cytogenetic comparisons were categorized as follows: same abnormal relapse clone $(6 / 18,33 \%)$, relapse clone with some similarities $(4,22 \%)$, relapse clone showing clonal evolution $(4,22 \%)$, and different relapse clone $(4,22 \%)$. Some cases were placed in the "relapse clone with some similarities" category because only targeted FISH analysis and not a full karyotype was performed at the time of relapse.

Flow cytometry data was available for a subset of specimens. Of the nine pre-transplant flow cytometry studies, three showed an increase in blasts and three showed an abnormal immunophenotype on blasts (heterogeneous/partial CD7 expression or homogenous expression of antigens/discrete cluster). The majority of relapse disease flow cytometry specimens $(15 / 19,79 \%)$ had increased blasts. In those cases without increased blasts, immunophenotypic abnormalities noted but not considered definitive included heterogenous or partial CD7 expression or homogenous expression of myeloid markers such as CD13 and CD33. A single pair of pre-transplant and relapse flow cytometry studies showed immunophenotypic similarities with partial CD7 expression in both.

\section{Early versus late relapse (Table 1)}

The median time to relapse after transplant was 6 months (range 2-82). Eleven patients had a late relapse, defined as $>180$ days ( 6 months) after transplant, with a median time to relapse of 15.4 months (range 6-81.6 months). The ten patients with early relapse had a median time to relapse of 3 months (range 2.2-5.8). Table 1 compares the patients with early versus late relapse.

To summarize, patients with late relapse had a younger median age at transplant and had a higher median bone marrow blast percentage at relapse. Donor source (umbilical cord blood versus non-umbilical cord blood), conditioning regimen (myeloablative versus nonmyeloablative), and IPSS risk score were not associated with timing of relapse, although the number of patients in each category was small. All three therapy-related MDS cases were late relapses, occurring 6, 19, and 
Table 1 Comparison of Hematologic and Morphologic Features Between Early and Late Relapse Patients

\begin{tabular}{|c|c|c|c|}
\hline & $\begin{array}{l}\text { Early Relapse (<6 months) } \\
n=10\end{array}$ & $\begin{array}{l}\text { Late Relapse (>6 months) } \\
n=11\end{array}$ & $p$-value \\
\hline Age at transplant, years, median (range) & $67(34-71)$ & $58(46-61)$ & 0.013 \\
\hline Gender, M:F & $5: 5$ & $6: 5$ & NS \\
\hline Time to relapse from transplant, months, median (range) & $3(2-6)$ & $15(6-82)$ & $<0.001$ \\
\hline \multicolumn{4}{|c|}{ Pre-transplant MDS specimen, peripheral blood and bone marrow characteristics } \\
\hline Hgb, g/dL, median(range) & $9.0(7.6-12.7)$ & $9.3(5.6-12.9)$ & NS \\
\hline MCV, fL, median (range) & $89(73-109)$ & $101(86-107)$ & 0.023 \\
\hline WBC, $\times 10^{9} / \mathrm{L}$, median (range) & $3.0(1.1-10.2)$ & $3.3(1-33)$ & NS \\
\hline ANC, $\times 10^{9} / \mathrm{L}$, median (range) & $0.9(0.1-7.8)$ & $1.1(0.4-24.1)$ & NS \\
\hline Platelets, $\times 10^{9} / L$, median (range) & $36(10-1242)$ & $89(5-206)$ & NS \\
\hline Blood blasts, \%, median (range) & $1(0-16)$ & $0.5(0-13)$ & NS \\
\hline Dysgranulopoiesis $^{\mathrm{a}}$ & $4 / 7$ & $3 / 8$ & NS \\
\hline Dyserythropoeisis $^{\mathrm{a}}$ & $5 / 7$ & $6 / 8$ & NS \\
\hline Dysmegakaryopoiesis $^{\mathrm{a}}$ & $4 / 6$ & $5 / 3$ & NS \\
\hline Ring sideroblasts $^{a}$ & $4 / 8$ & $4 / 9$ & NS \\
\hline Marrow cellularity, \%, median (range) & $90(15-95)$ & $43(30-95)$ & 0.075 \\
\hline Bone marrow blasts, \%, median (range) & $4(1-19)$ & $7(2-14)$ & NS \\
\hline Increased marrow blasts (>5\%) & $7 / 10(70 \%)$ & $8 / 11(72 \%)$ & NS \\
\hline \multicolumn{4}{|c|}{ Relapse myeloid neoplasm specimen, peripheral blood and bone marrow characteristics } \\
\hline Hgb, g/dL, median(range) & $10.0(8.6-12.2)$ & $9.8(8.1-13)$ & NS \\
\hline MCV, fL, median (range) & $88(80-105)$ & $99(87-113)$ & 0.051 \\
\hline $\mathrm{WBC}, \times 10^{9} / \mathrm{L}$, median (range) & $2.2(0.6-7.6)$ & $2.5(1.5-3.4)$ & NS \\
\hline ANC, $\times 10^{9} / \mathrm{L}$, median (range) & $1.3(0.2-5.9)$ & $1.2(0.2-2.5)$ & NS \\
\hline Platelets, $\times 10^{9} / \mathrm{L}$, median (range) & $20(7-128)$ & $66(10-189)$ & 0.061 \\
\hline Blood blasts, \%, median (range) & $0.3(0-15)$ & $2(0-38)$ & NS \\
\hline Dysgranulopoiesis ${ }^{a}$ & $3 / 9$ & $2 / 11$ & NS \\
\hline Dyserythropoeisis $^{a}$ & $5 / 9$ & $6 / 11$ & NS \\
\hline Dysmegakaryopoiesis $^{a}$ & $7 / 8: 5 / 8$ & $6 / 10: 3 / 10$ & NS \\
\hline Ring sideroblasts $^{a}$ & $6 / 6$ & $1 / 3$ & 0.033 \\
\hline Marrow cellularity, \%, median (range) & $48(20-90)$ & $45(10-90)$ & NS \\
\hline Bone marrow blasts, $\%$, median (range) & $5(0.2-30)$ & $14(4-51)$ & 0.029 \\
\hline Increased marrow blasts (>5\%) & $5 / 10(50 \%)$ & 10/11 (91\%) & 0.063 \\
\hline
\end{tabular}

Bold indicates statistical significance

${ }^{a}$ Any degree of dysplasia $\geq 10 \%$ of a lineage. See text (materials and methods section) for specific dysplastic features included for this study; " $n$ " for dysplasia and ring sideroblast evaluation varies for each category depending on the slides and number of precursors in each lineage available for review

49 months after transplant. Dysplasia characteristics in the pre-transplant or relapse specimens did not show a statistically significant association with timing of relapse, nor did the cytogenetic comparison. There was no association between relapse as overt AML (>20\% blasts) and timing of relapse.

\section{Pre-relapse marrow evaluation}

Post-transplant bone marrow biopsy specimens evaluated at our institution up to and including the relapse specimen are presented in Tables 2 and 3 (divided into early and late relapse cases) including morphologic interpretation, engraftment results, and cytogenetics results. There was no significant association between a morphologic interpretation of indeterminate and subsequent morphologic interpretation of relapse (6 of 15 specimens interpreted as indeterminate and 14 of 48 specimens interpreted as negative had morphologic relapse in the subsequent marrow biopsy, $p=0.528$ ).

For this study, we retrospectively reviewed, blinded to the original interpretation and time-point, slides from the bone marrow biopsy immediately prior to the relapse specimen. The morphologic conclusion at the time of original interpretation and at the time of review for this 


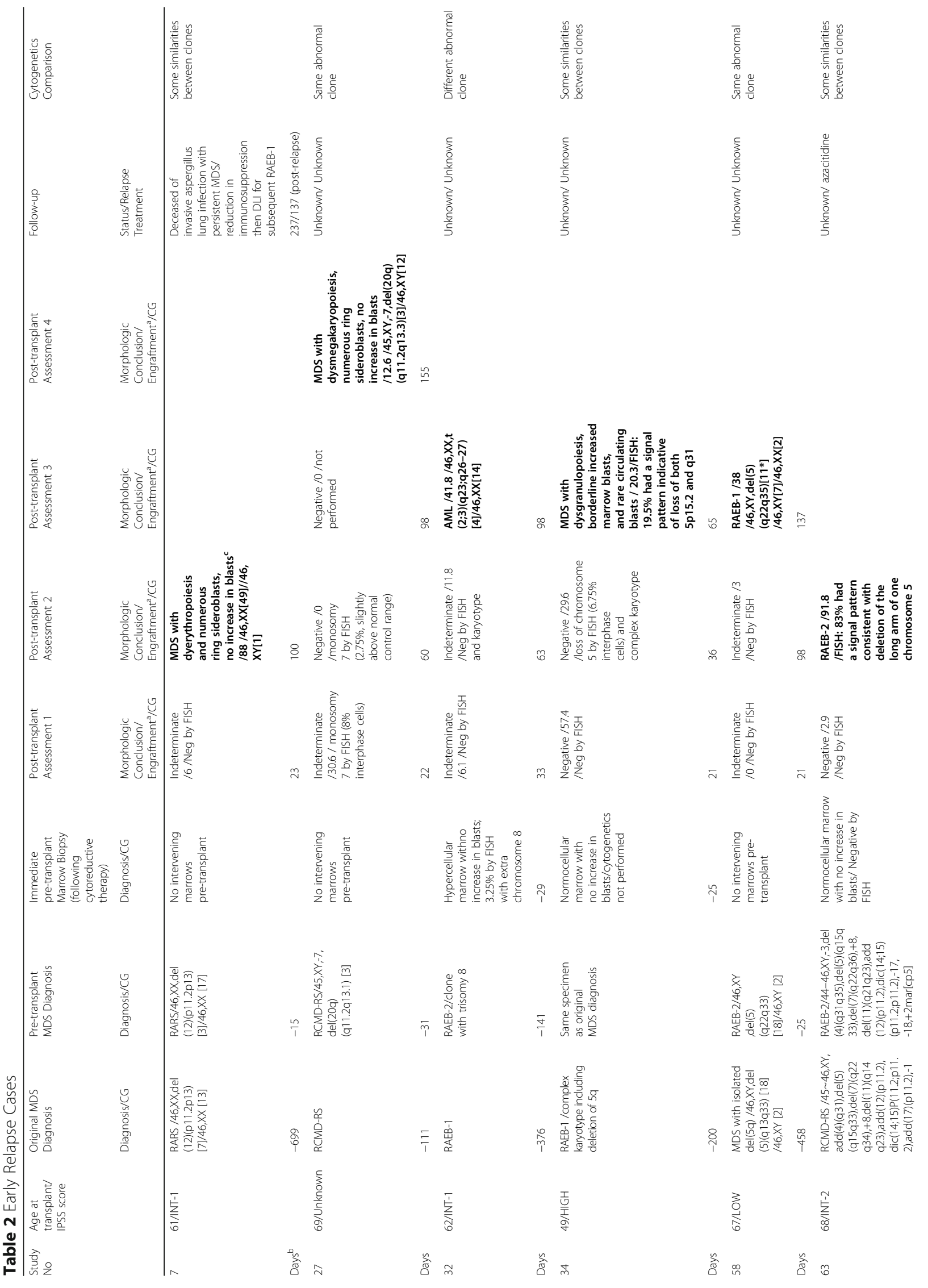




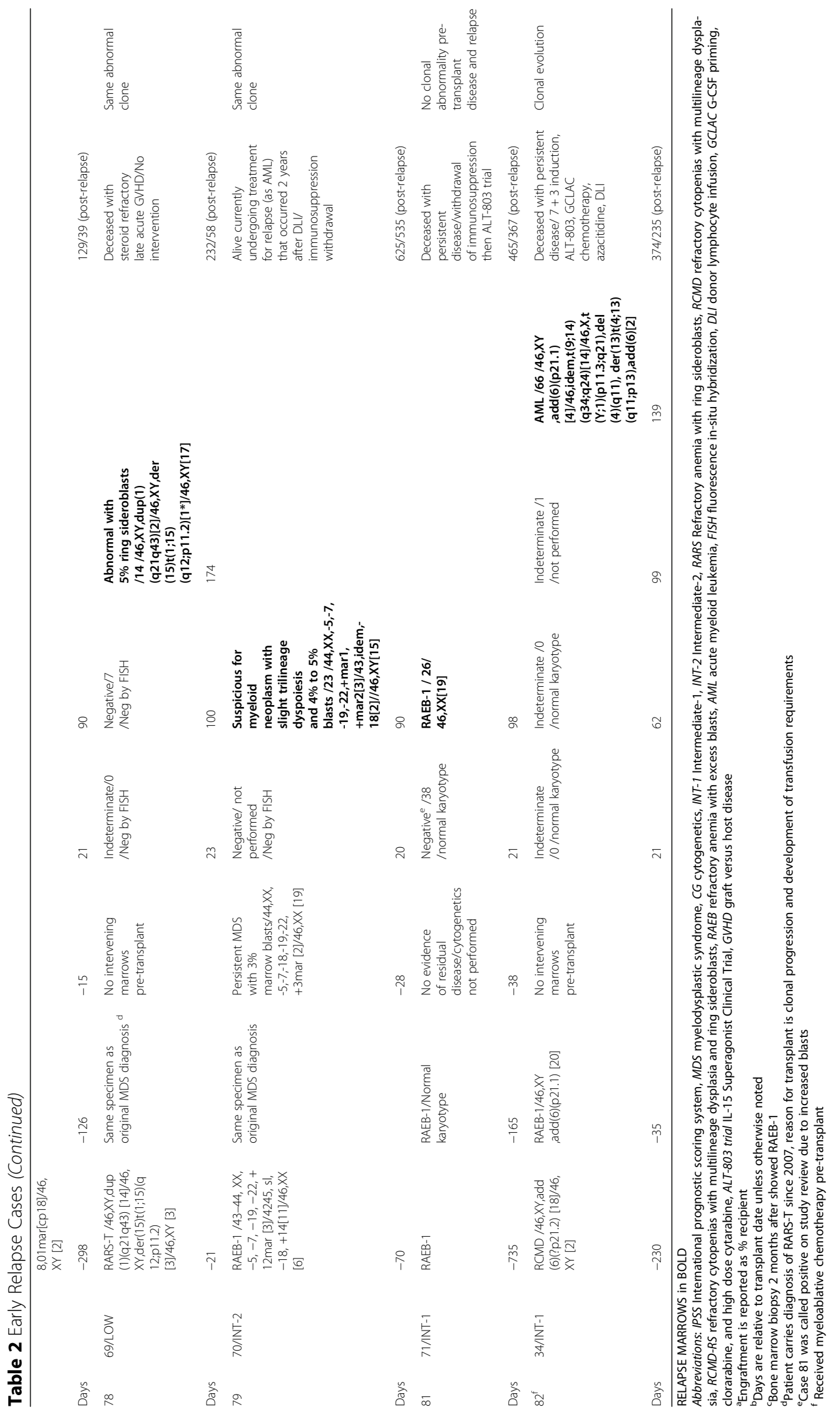




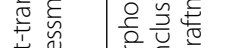

言合

잉

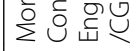

$\stackrel{m}{n}$

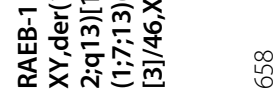

Courville et al. BMC Clinical Pathology (2017) 17:28

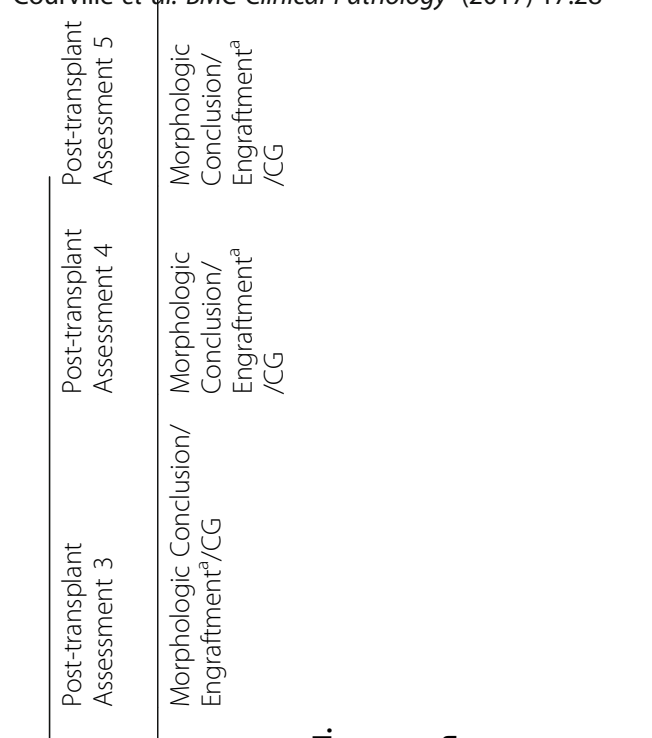

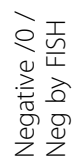

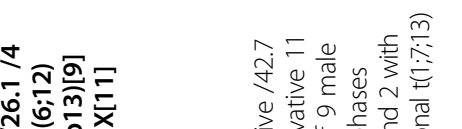

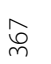

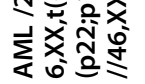
웅

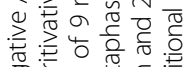

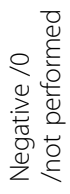

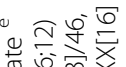

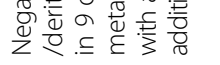

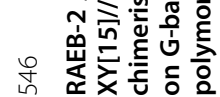

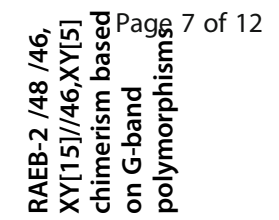

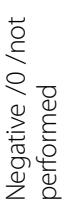

o

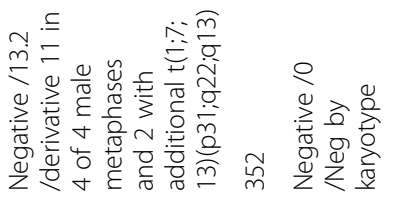

œ $\quad \frac{0}{2} \stackrel{0}{2}$

$\stackrel{-}{\infty}$
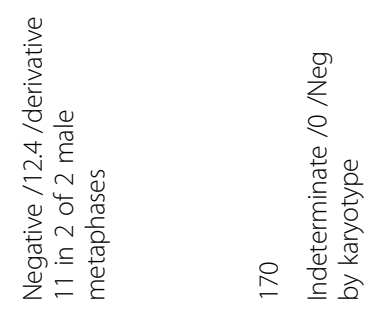

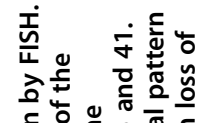

满

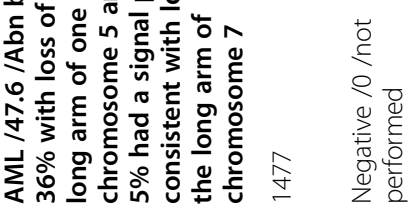
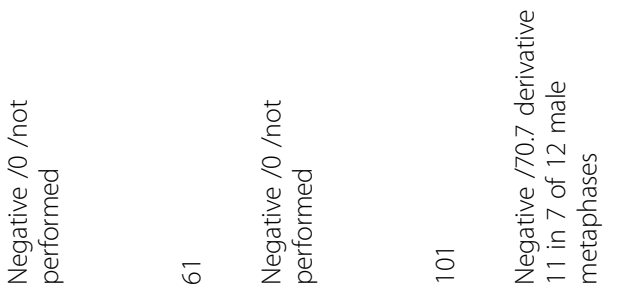

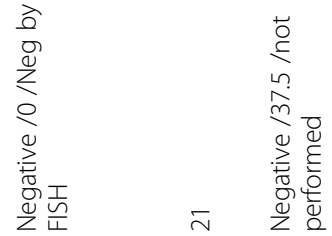

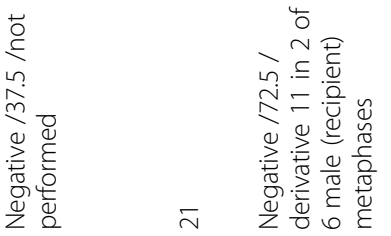

๑ 苞这

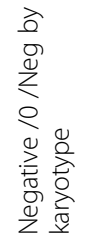

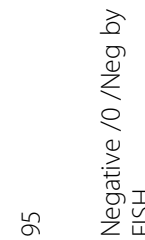

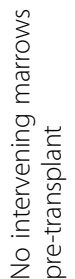

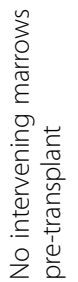

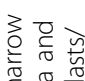

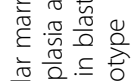

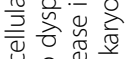

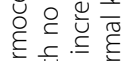

v

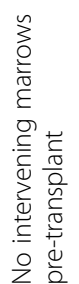

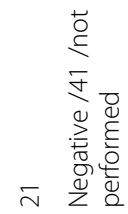

훙 $\frac{\bar{y}}{0} \times$

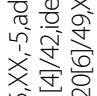

过 们

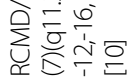

亲过爻

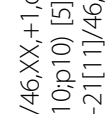

₹ $\sum_{\substack{0 \\ 0}}^{0}$

紊文爻

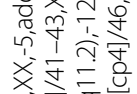

爻过容莙

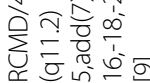

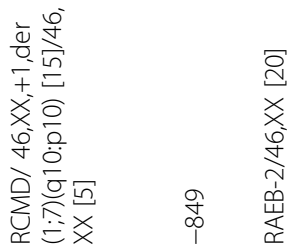

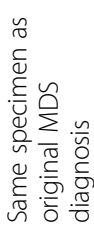

产 $\frac{\bar{m}}{\dot{0}}$

这遂遂

岀商过
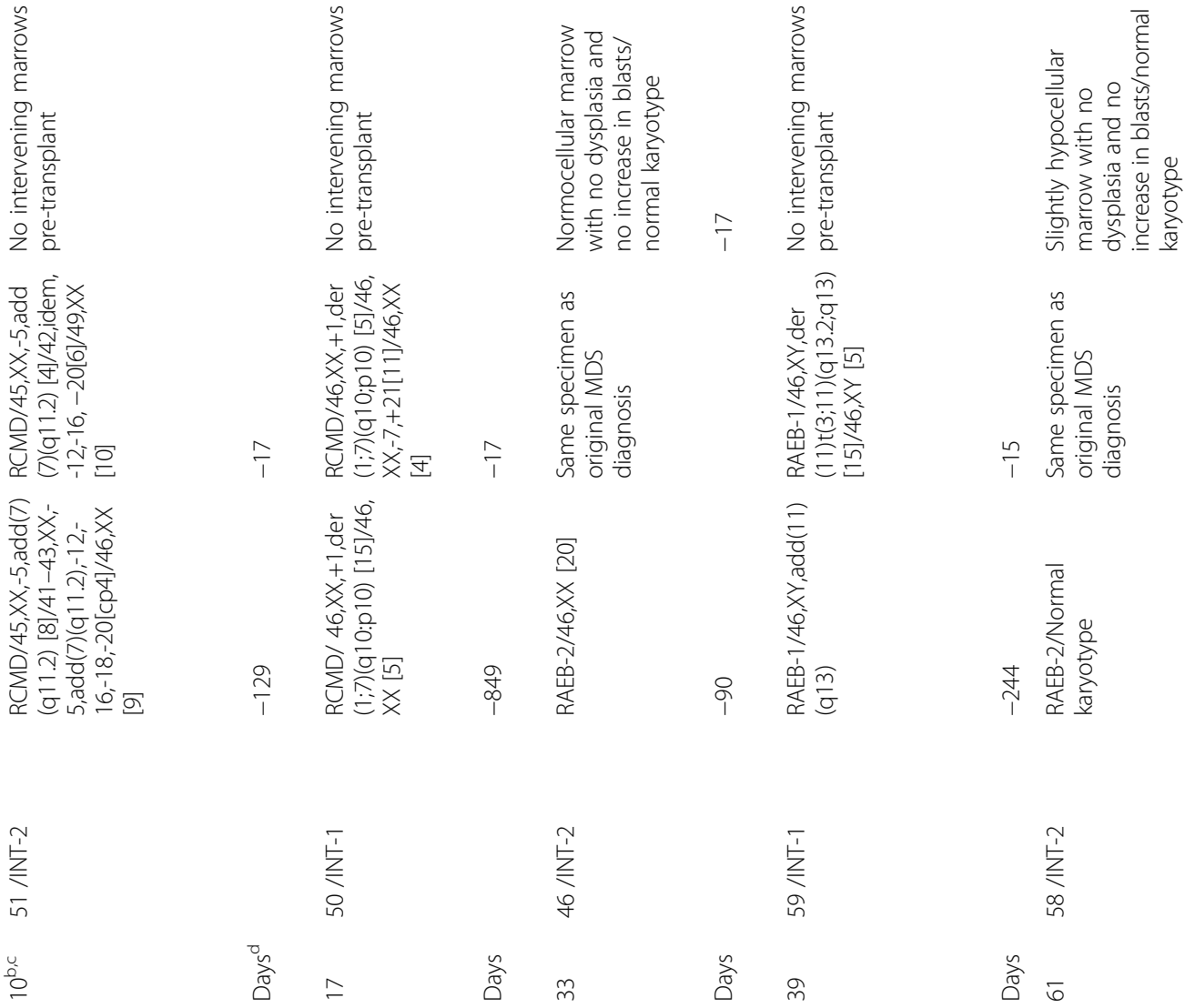

$\stackrel{\bar{\Sigma}}{\stackrel{\bar{s}}{2}}$

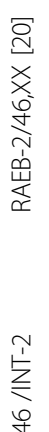

产

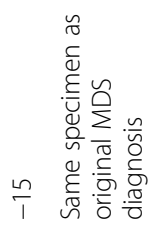

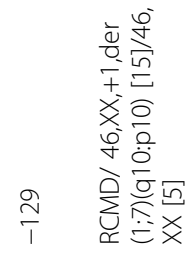

椧

ลิ

๙ิ̊

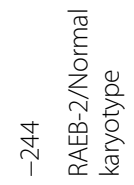

or

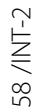

ลิ 


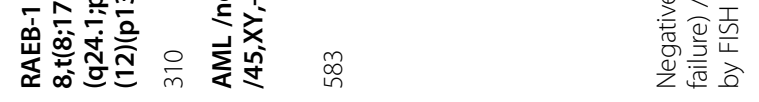

Courville et al. BMC Clinical Pathoßgy (2017) 17:28

Page 8 of 12

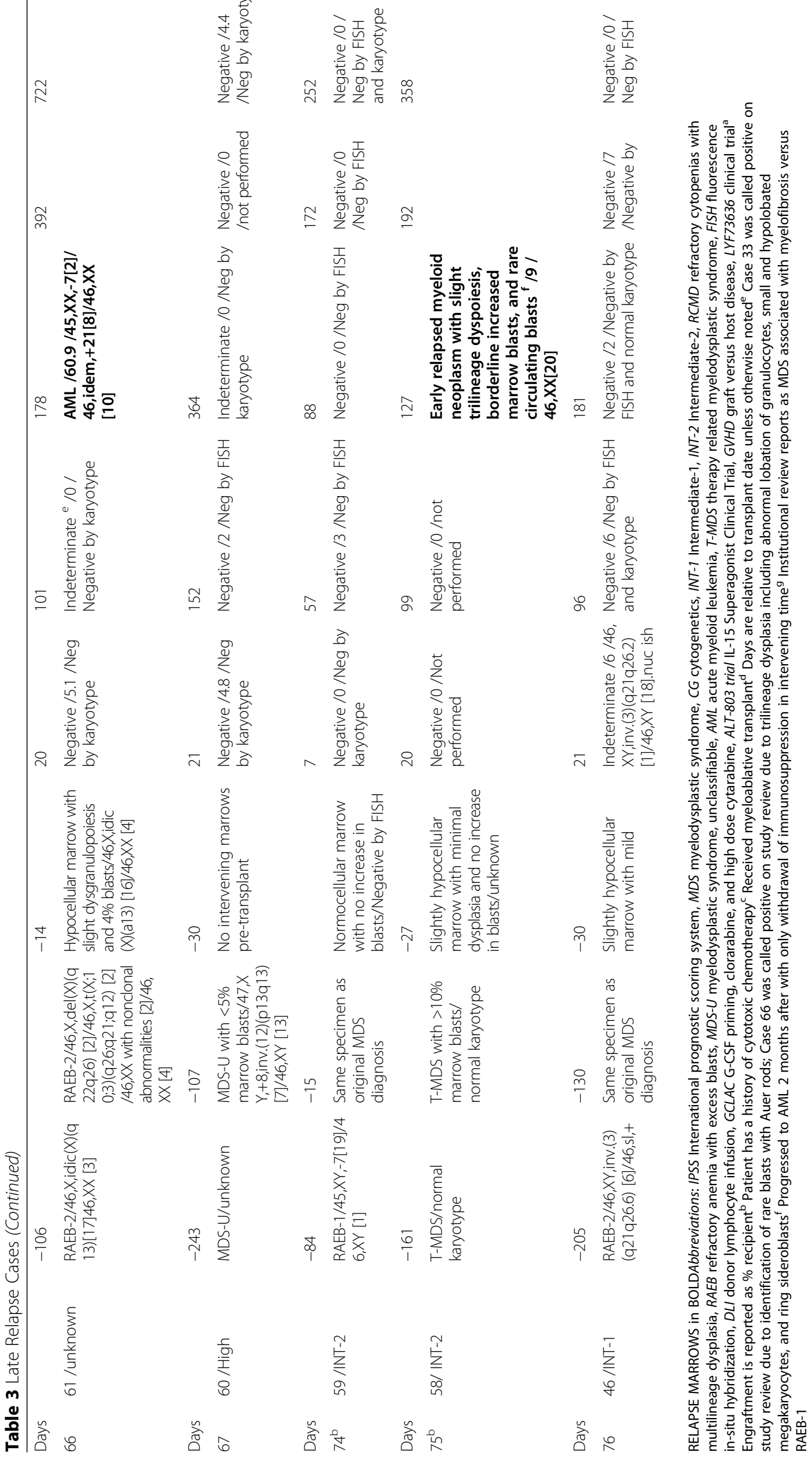


study varied for nine of the cases, mainly between negative and indeterminate (six cases). Three cases (cases 81, 66, and 33) were re-interpreted as positive for myeloid neoplasm. Case 81 had dysplastic granulocytes, rare ring sideroblasts, atypical monocytes, and increased blasts. Case 66 showed trilineage dysplasia including abnormal lobation in granulocytes, small and hypolobate megakaryocytes, and ring sideroblasts. Case 33 lacked dysplasia and did not have an increase in blasts; however rare blasts with Auer rods were seen in the peripheral blood and bone marrow aspirate slides.

\section{Outcomes post relapse}

Four of the five patients with early relapse and known follow-up were deceased, with a median survival of 306 days post-transplant (range 232 to 465 days) and 186 days post-relapse (range 58 to 367 days). The one patient (Case 79) alive at last follow-up of 625 days posttransplant/535 days post-relapse was treated for relapse with withdrawal of immunosuppression and donor lymphocyte infusion and achieved complete remission for approximately 2 years followed by relapse as AML for which she is undergoing treatment.

Eight of the ten patients with late relapse and known follow-up were deceased, with a median survival of 608 days post-transplant (range 298-2604 days) and 120 days post-relapse (range 40-583 days). Treatment protocols following disease relapse varied and are detailed in Table 3. Two patients were alive at last follow-up, 539 and 1892 days post-relapse, both in complete remission. Case 33 was 46 years old at the time of transplant and relapsed with AML for which she was treated with induction chemotherapy followed by HaploNK therapy with transplant. Case 76, also 46 years old at the time of transplant, had graft failure within a year of transplant for which he received a stem cell boost; subsequent relapsed disease (characterized by dyspoiesis in two lineages and $4 \%$ to $7 \%$ blasts) was treated with a second allogeneic sibling transplant.

\section{Discussion}

Our study highlights the challenges of predicting MDS relapse post allogeneic HCT. Our patient cohort showed varied morphologic findings between pre-transplant disease and relapse with a large percentage of patients relapsing with a higher grade/higher blast MDS or frank AML. We found the presence of pre-transplant megakaryocyte dysplasia to correspond with the presence of megakaryocyte dysplasia in the relapse specimen but no consistency with erythroid or granulocytic dysplasia between pre- and post-transplant specimens. Similar to previous studies [11], cytogenetic abnormalities in the pre-transplant and relapse disease showed variability, including substantial proportions with clonal evolution pre-transplant to relapse or emergence of a previously undetected/undetectable clone. While the available flow cytometric data was too sparse in our cohort, studies on the immunophenotype of neoplastic blasts and maturing myeloid lineage cells in MDS pre-transplant and at relapse using modern and reproducible parameters (such as outlined by the European LeukemiaNet Working Group [12]) is needed. Our data highlight the need for additional more objective and quantitative measures of disease such as genetic profiling for common MDS mutations [13-15] for assessment of efficacy of interventions for relapse/impending relapse, such as that presented by Woo et al. [16].

Previous work by our group focusing on patients transplanted for MDS evaluated features of the marrow in the immediate pre-transplant biopsy, including blast percentage and the percentage of cytogenetically abnormal cells, and correlated with outcomes (survival and relapse). The Trottier study [17] evaluated patients from 1995 to the end of 2012 and only included those patients with abnormal cytogenetics in the diagnostic MDS sample. In contrast, in our current study, we performed a detailed retrospective evaluation of blood and marrow features prior to any preparative or cytoreductive therapy performed in anticipation of transplant and contrasted to blood and marrow features of the relapse specimen. In addition, our study includes only those patients with known relapse and we include all relapse patients and not those only with abnormal cytogenetics at MDS diagnosis. In reviewing the cases in common, 15 of the cases in the current analysis are in common with the Trottier report, which included 82 MDS patients. The current work expands on prior work in an attempt to identify features of impending relapse.

Our relapse patterns were consistent with the literature that describes the majority of relapses after allogeneic transplant for MDS occurring within the first year post transplant. Thirteen (62\%) of the relapses in our cohort occurred within a year of transplant. There was a near even division of our patient cohort between those that relapsed before and after 6 months, defined as early and late relapse for this study. We found no specific features that differed between patients with late versus early relapse, with the exception of age and marrow blast percentage, likely correlating with myeloablative conditioning. Perhaps counter-intuitive to the usual poor prognosis attributed to therapy-related myeloid neoplasms, all three t-MDS cases were late relapses occurring 6, 19, and 49 months after transplant. Eighteen months has been used previously in the literature to define late versus early relapse [11]. In our cohort, the five patients who relapsed more than 18 months after transplant (patients 10, 17, 39, 61, and 74) did not show distinguishing features. 
Relapse and impending relapse are difficult to define in the context of post-allogeneic stem cell transplant for MDS, particularly for low-grade MDS. Relapsed MDS does not always have increased blasts and dysplasia alone may be the only feature suggesting impending relapse. Unfortunately, the specificity of dysplasia in the post-transplant setting is low, even with published criteria for the definition and enumeration of ring sideroblasts and morphologic dysplasia [18, 19]. Dyspoiesis is described following bone marrow transplant in the erythroid lineage (including ring sideroblasts), granulocytic lineage, and megakaryocytes [20-22]. Granulocytic dysplasia can be seen in the context of immunosuppressive medication such as tacrolimus or mycophenylate mofetil [23]. Borderline increased blasts can also be seen with recombinant growth factor therapy (G-CSF and GM-CSF) administration and early robust marrow regeneration. In addition, a graft-versus leukemia effect may suppress persistent disease/impending relapse without additional intervention. Due to these factors, there is variability in the interpretation and clinical significance assigned to morphologic dysplasia in the post-transplant context. Our morphologic re-review of pre-relapse biopsies emphasizes the inter-observer variability in interpretation of dysplasia, with a different morphologic conclusion from the original interpretation in nine cases. Thus additional criteria beyond morphologic dysplasia are needed to help identify impending relapse.

Cytogenetic analysis is a valuable tool for detecting impending relapse. However, as a subset of MDS lack a cytogenetic abnormality and sampling may be a source of false negatives, results of cytogenetic analysis do not always yield the final answer. In addition, the relapse clonal abnormality may vary from the pre-transplant disease limiting the utility of directed FISH analysis. Genetic mutation analysis holds promise in detection of impending relapse, even in cases without cytogenetic abnormalities, although sampling and limits of detection are potentials for falsenegatives and clonal heterogeneity within an MDS [24, 25] can create complexity in interpretation. Engraftment/chimerism studies can aid in identification of impending relapse; however, donor-cell derived MDS after allogeneic stem cell transplant [26] is a well described entity.

\section{Conclusions}

A pragmatic approach to detection of MDS relapse and more importantly impending relapse following transplant is needed. Such an approach includes incorporation of morphologic, cytogenetic, and molecular data (including engraftment/chimerism studies). As with the initial diagnosis of MDS [27], it may take multiple sequential biopsies to make a definitive diagnosis of relapsed MDS. Detection of impending relapse is more difficult in cases with normal cytogenetics as morphologic features of dysplasia overlap with post-transplant changes and the dysplastic lineage(s) of the relapse disease may not correspond to those of the pre-transplant disease; molecular mutational analysis may prove very beneficial in these cases. While the appropriate intervention(s) for relapse and impending relapse is not well established, identifying definitive impending relapse and early relapse may allow for targeted interventions that may prevent a full blown relapse and improve patient survival. The pathologist's role in diagnosis of impending relapse/relapse includes not only accurate morphologic identification of dysplasia and blast percentage, but an appreciation and knowledge of the multimodal approach to an MDS relapse diagnosis.

\section{Abbreviations \\ AML: Acute myeloid leukemia; HCT: Hematopoietic cell transplant; IPSS: International prognostic scoring system; MDS: Myelodysplastic syndrome; RAEB: Refractory anemia with excess blasts; WHO: World Health Organization}

\section{Acknowledgements}

Not applicable

\section{Funding}

Research reported in this publication was supported by NIH grant P30 CA77598 utilizing the Biostatistics and Bioinformatics Core shared resource of the Masonic Cancer Center, University of Minnesota and by the National Center for Advancing Translational Sciences of the National Institutes of Health Award Number UL1TR000114. The content is solely the responsibility of the authors and does not necessarily represent the official views of the National Institutes of Health.

\section{Availability of data and materials}

The datasets used and/or analyzed during the current study are available from the corresponding author on reasonable request.

\section{Authors' contributions}

EC devised the study design, collected and analyzed data, and wrote the manuscript; MG collected and analyzed data and edited the manuscript; CU collected data and edited the manuscript; SY collected data and edited the manuscript; EW collected data and wrote the manuscript. All authors have read and approved the manuscript.

\section{Ethics approval and consent to participate}

This study received approval from the University of Minnesota Institutional Review Board. As part of the approval process, the University of Minnesota determined that consent was not required.

Consent for publication

Not applicable.

Competing interests

The authors have no relevant competing interests or financial disclosures.

\section{Publisher's Note}

Springer Nature remains neutral with regard to jurisdictional claims in published maps and institutional affiliations.

\section{Author details}

'Department of Laboratory Medicine and Pathology, University of Minnesota, 420 Delaware St SE, MMC 609, Minneapolis, MN 55455, USA. ${ }^{2}$ Division of Hematology, Oncology, and Transplantation, Department of Medicine, University of Minnesota, Minneapolis, MN, USA. 
Received: 12 September 2017 Accepted: 15 December 2017

Published online: 28 December 2017

\section{References}

1. Arber DA, Orazi A, Hasserjian R, Thiele J, Borowitz MJ, Le Beau MM, Bloomfield CD, Cazzola M, Vardiman JW. The 2016 revision to the World Health Organization classification of myeloid neoplasms and acute leukemia. Blood. 2016;127(20):2391-405.

2. Fenaux P, Ades L. How we treat lower-risk myelodysplastic syndromes. Blood. 2013;121(21):4280-6.

3. Sekeres MA, Cutler C. How we treat higher-risk myelodysplastic syndromes. Blood. 2014;123(6):829-36.

4. Greenberg P, Cox C, LeBeau MM, Fenaux P, Morel P, Sanz G, Sanz M, Vallespi T, Hamblin T, Oscier D, et al. International scoring system for evaluating prognosis in myelodysplastic syndromes. Blood. 1997:89(6):2079-88

5. Greenberg PL, Tuechler H, Schanz J, Sanz G, Garcia-Manero G, Sole F, Bennett JM, Bowen D, Fenaux P, Dreyfus F, et al. Revised international prognostic scoring system for myelodysplastic syndromes. Blood. 2012; 120(12):2454-65

6. Kindwall-Keller T, Isola LM. The evolution of hematopoietic SCT in myelodysplastic syndrome. Bone Marrow Transplant. 2009;43(8):597-609.

7. Christopeit M, Kroger N, Haferlach T, Bacher U. Relapse assessment following allogeneic SCT in patients with MDS and AML. Ann Hematol. 2014:93(7):1097-110.

8. Warlick ED, Cioc A, Defor T, Dolan M, Weisdorf D. Allogeneic stem cell transplantation for adults with myelodysplastic syndromes: importance of pretransplant disease burden. Biol Blood Marrow Transplant. 2009;15(1):30-8.

9. Weinberg OK, Pozdnyakova O, Campigotto F, DeAngelo DJ, Stone RM, Neuberg D, Hasserjian RP. Reproducibility and prognostic significance of morphologic dysplasia in de novo acute myeloid leukemia. Mod Pathol. 2015;28(7):965-76.

10. Thyagarajan B, Young S, Floodman S, Peterson R, Wang X. Systematic analysis of interference due to stutter in estimating chimerism following hematopoietic cell transplantation. J Clin Lab Anal. 2009;23(5):308-13.

11. Yeung CC, Gerds AT, Fang M, Scott BL, Flowers ME, Gooley T, Deeg HJ. Relapse after Allogeneic hematopoietic cell transplantation for Myelodysplastic syndromes: analysis of late relapse using comparative Karyotype and chromosome genome Array testing. Biol Blood Marrow Transplant. 2015;21(9):1565-75.

12. Westers TM, Ireland R, Kern W, Alhan C, Balleisen JS, Bettelheim P, Burbury K, Cullen M, Cutler JA, Della Porta MG, et al. Standardization of flow cytometry in myelodysplastic syndromes: a report from an international consortium and the European LeukemiaNet working group. Leukemia. 2012;26(7):1730-41.

13. Bejar R, Levine R, Ebert BL. Unraveling the molecular pathophysiology of myelodysplastic syndromes. J Clin Oncol. 2011;29(5):504-15.

14. Bejar R, Stevenson K, Abdel-Wahab O, Galili N, Nilsson B, Garcia-Manero G, Kantarjian H, Raza A, Levine RL, Neuberg D, et al. Clinical effect of point mutations in myelodysplastic syndromes. N Engl J Med. 2011:364(26):2496-506.

15. Ciabatti E, Valetto A, Bertini V, Ferreri Ml, Guazzelli A, Grassi S, Guerrini F, Petrini I, Metelli MR, Caligo MA, et al. Myelodysplastic syndromes: advantages of a combined cytogenetic and molecular diagnostic workup. Oncotarget. 2017;8(45):79188-9200.

16. Woo J, Howard NP, Storer BE, Fang M, Yeung CC, Scott BL, Deeg HJ. Mutational analysis in serial marrow samples during azacitidine treatment in patients with post-transplant relapse of acute myeloid leukemia or myelodysplastic syndromes. Haematologica. 2017;102(6):e216-8.

17. Trottier BJ, Sachs Z, DeFor TE, Shune L, Dolan M, Weisdorf DJ, Ustun C, Warlick ED. Novel disease burden assessment predicts allogeneic transplantation outcomes in myelodysplastic syndrome. Bone Marrow Transplant. 2016;51(2):199-204.

18. Brunning RD, Orazi A, Germing U, Le Beau MM, Porwit A, Baumann I, Vardiman JW, Hellstrom-Lindberg E. Myelodysplastic syndromes/neoplasms, overview. In: Swerdlow SH, Campo E, Harris NL, Jaffe ES, Pileri SA, Stein H, Thiele J, Vardiman JW, editors. WHO classification of tumours of haematopoietic and lymphoid tissues. 4th ed. Lyon: IARC; 2008. p. 88-93.

19. Mufti GJ, Bennett JM, Goasguen J, Bain BJ, Baumann I, Brunning R, Cazzola M, Fenaux P, Germing U, Hellstrom-Lindberg E, et al. Diagnosis and classification of myelodysplastic syndrome: international working group on morphology of myelodysplastic syndrome (IWGM-MDS) consensus proposals for the definition and enumeration of myeloblasts and ring sideroblasts. Haematologica. 2008;93(11):1712-7.
20. Reichard KK, Foucar K. Bone marrow morphologic changes after chemotherapy and stem cell transplantation. In: Orazi A, Foucar K, Knowles D, Weiss LM, editors. Knowles' Neoplastic Hematopathology. 3rd ed. Philadelphia, PA: Lippincott Williams \& Wilkins; 2014.

21. van Marion AM, Thiele J, Kvasnicka HM, van den Tweel JG. Morphology of the bone marrow after stem cell transplantation. Histopathology. 2006;48(4):329-42.

22. van den Berg H, Kluin PM, Vossen JM. Early reconstitution of haematopoiesis after allogeneic bone marrow transplantation: a prospective histopathological study of bone marrow biopsy specimens. J Clin Pathol. 1990;43(5):365-9.

23. Bain BJ, Clark DM, Wilkins BS. Bone marrow pathology. 4th ed. West Sussex: Wiley-Blackwell; 2010.

24. Bejar $\mathrm{R}$, Abdel-Wahab $\mathrm{O}$. The importance of subclonal genetic events in MDS. Blood. 2013;122(22):3550-1.

25. Papaemmanuil E, Gerstung M, Malcovati L, Tauro S, Gundem G, Van Loo P, Yoon CJ, Ellis P, Wedge DC, Pellagatti A, et al. Clinical and biological implications of driver mutations in myelodysplastic syndromes. Blood. 2013; 122(22):3616-27. quiz 3699

26. Dietz AC, DeFor TE, Brunstein CG, Wagner JE. Donor-derived myelodysplastic syndrome and acute leukaemia after allogeneic haematopoietic stem cell transplantation: incidence, natural history and treatment response. Br J Haematol. 2014;166(2):209-12.

27. Malcovati L, Hellstrom-Lindberg E, Bowen D, Ades L, Cermak J, Del Canizo C, Della Porta MG, Fenaux P, Gattermann N, Germing U, et al. Diagnosis and treatment of primary myelodysplastic syndromes in adults: recommendations from the European LeukemiaNet. Blood. 2013;122(17):2943-64.

\section{Submit your next manuscript to BioMed Central and we will help you at every step:}

- We accept pre-submission inquiries

- Our selector tool helps you to find the most relevant journal

- We provide round the clock customer support

- Convenient online submission

- Thorough peer review

- Inclusion in PubMed and all major indexing services

- Maximum visibility for your research

Submit your manuscript at www.biomedcentral.com/submit
) Biomed Central 